\title{
PROPRIEDADES FÍSICAS E MECÂNICAS DE LATOSSOLOS EM DIFERENTES MANEJOS FLORESTAIS ${ }^{1}$
}

\author{
Physical and mechanical properties of Latosols in different forest management ${ }^{1}$
}

\author{
Arystides Resende Silva², Moacir de Souza Dias Junior ${ }^{3}$, Fernando Palha Leite ${ }^{4}$
}

\begin{abstract}
RESUMO
Com a intensa mecanização das operações florestais, a compactação do solo, causada pelo tráfego, pode ser limitante para a obtenção de maiores produtividades.Objetivou-se, neste estudo, avaliar as alterações em algumas propriedades físicas e mecânicas dos seguintes Latossolos: a) Latossolo Vermelho-Amarelo devido a distintas intensidades de tráfego e carga de um Forwarder na Região de Santa Maria de Itabira, MG; b) Latossolo Amarelo e um Latossolo Vermelho devido à distintas intensidades de tráfego de um Forwarder sobre diferentes condições de disposição de resíduos nas Regiões de Belo Oriente e São João Evangelista, MG. Na Região de Santa Maria de Itabira, foram coletadas, nas profundidades de 0-3 e 10-13 cm, 10 amostras indeformadas onde não houve tráfego e na linha de tráfego o Forwarder percorreu 2, 4 e 8 vezes e no local esse passou 4 vezes com 1/3, 2/3 e $3 / 3$ de sua carga. Nas Regiões de Belo Oriente e São João Evangelista, foram coletadas 20 amostras indeformadas na linha de tráfego e o Forwarder trafegou 2 e 8 vezes, em quatro condições de disposição de resíduos. As amostras indeformadas foram utilizadas nos ensaios de compressão uniaxial. Determinaram-se também as características físicas e químicas dos solos. Todas as intensidades tráfego e carga do Forwarder na Região de Santa Maria de Itabira causaram compactação no solo, principalmente na profundidade de $10-13 \mathrm{~cm}$. Nas Regiões de Belo Oriente e São João Evangelista, o número de passadas do Forwarder igual a 8 foi a que causou maior compactação do solo, sendo essa minimizada nas condições de resíduos galhada mais casca e galhada.
\end{abstract}

Termos para indexação: Compactação do solo, densidade do solo, volume total de poros, pressão de preconsolidação, mecanização florestal, Eucalyptus spp.

\section{ABSTRACT}

With the intense mechanization of forest operations, soil compaction, caused by the traffic, may limit high productivities. The objective of this study was to evaluate the alterations in the physical and mechanical properties of the following Latosol: a) RedYellow Latosol due to different traffic intensities and load of a Forwarder in the Santa Maria de Itabira region - MG; b) Yellow Latosol and a Red Latosol due to different traffic intensities of a Forwarder on different residue disposition in the Belo Oriente and São João Evangelista regions, MG. In the Santa Maria de Itabira region, it were collected at 0-3 and 10-13 cm depths, 10 undisturbed soil samples with no traffic and where the Forwarder trafficked 2, 4 and 8 times and in the place where the Forwarder trafficked 4 times with 1/3, 2/3 and 3/3 of its load. In the Belo Oriente and São João Evangelista regions, 20 undisturbed soils samples were collected in the traffic line where the Forwarder trafficked 2 and 8 times, in four conditions of residue disposition. The undisturbed soil samples were used in the uniaxial compression. It was also determined, physical and chermical characteristics of the soils. All the traffic intensities and load of the Forwarder in the Santa Maria de Itabira region caused soil compaction mainly at 10-13 cm depth. I the Belo Oriente and São João Evangelista regions, the number of passes of the Forwarder equal to 8 caused greater compaction of the soil, being minimized, in the residue conditions of Brushwood plus Bark and Brushwood.

Index terms: Soil compaction, soil density, total porosity, preconsolidation pressure, forest mechanization, Eucalyptus spp.

(Recebido em 21 de julho de 2009 e aprovado em 22 de março de 2010)

\section{INTRODUÇÃO}

A necessidade de realizar a colheita florestal em grandes áreas tem levado a um aumento na utilização de máquinas. As pressões exercidas por essas máquinas, durante as operações de colheita florestal, podem aplicar altas pressões sobre o solo (Horn et al., 2004), com consequentes alterações das propriedades físicas e mecânicas do solo, afetando a sua estrutura e resultando na sua compactação (Dias Júnior et al., 2005, Silva et al., 2007).

Os efeitos prejudiciais da compactação consistem em mudanças nas propriedades físicas do solo (Lebert \& Horn, 1991; Dias Júnior \& Pierce, 1996, 2005; Marchão et al., 2007; Suzuki et al., 2007: Modolo et al ., 2008), relacionadas

${ }^{1}$ Parte da Tese de Doutorado do primeiro autor, apresentado ao DCS - UFLA. Projeto financiado pela Celulose Nipo-Brasileira S.A (CENIBRA) e pela CAPES

${ }^{2}$ Empresa Brasileira de Pesquisa Agropecuária/Embrapa - Amazônia Oriental - Travessa Doutor Enéas Pinheiro - s/n - Bairro Marco - Cx. P. 48 66095-100 - Belém, PA - arystides@cpatu.embrapa.br

3Universidade Federal de Lavras/UFLA - Departamento de Ciência do Solo/DCS - Lavras, MG

${ }^{4}$ Celulose Nipo Brasileira S/A - Belo Oriente, MG

Ciênc. agrotec., Lavras, v. 34, n. 6, p. 1483-1491, nov./dez., 2010 
com seu espaço poroso, estrutura, permeabilidade ao ar e água (Hanza \& Anderson, 2005; Ajayui et al., 2009), com reflexo no desenvolvimento do sistema radicular (Marchão et al., 2007), com isso ocorrendo perda da sustentabilidade do processo de produção (Reichert et al., 2009).

A compreensão e a quantificação dos impactos causados pela compactação têm sido uma constante preocupação dos pesquisadores quando se considera a crescente mecanização associada ao aumento nas capacidades operacionais das máquinas, a intensidade de tráfego nas áreas (Hanza \& Anderson, 2005; Raper, 2005; Silva et al., 2007; Schaffer et al., 2008) e, principalmente, quando essas operações são realizadas em condições inadequadas de umidade do solo (Dias Júnior et al., 2005; Raper, 2005; Silva et al., 2007).

A quantificação da compactação do solo pode ser feita por meio do ensaio de compressão uniaxial, do qual obtém-se a curva de compressão do solo (Dias Júnior \& Pirce, 1996) e a partir dessa, a pressão de preconsolidação $\left(\sigma_{\mathrm{p}}\right)$ que é uma medida da capacidade de suporte de carga dos solos, e um indicador da sustentabilidade de sua estrutura (Dias Júnior \& Pierce, 1996; Dias Júnior et al., 2008). Assim sendo, o entendimento do comportamento compressivo do solo através das curvas de compressão adquire grande importância em razão de serem a base para a determinação da pressão de preconsolidação.

Objetivou-se, neste estudo, avaliar as alterações de algumas propriedades físicas e mecânicas dos seguintes Latossolos: a) Latossolo Vermelho-Amarelo, devido a diferentes intensidades de tráfego e carga de um Forwarder na Região de Santa Maria de Itabira, MG e b) Latossolo Amarelo e de um Latossolo Vermelho devido a diferentes intensidades de tráfego de um Forwarder sobre diferentes condições de disposição de resíduos, nas Regiões de Belo Oriente e São João Evangelista, MG.

\section{MATERIAL E MÉTODOS}

Para avaliar as alterações nas propriedades físicas e mecânicas do solo devido a diferentes manejos florestais, dois estudos foram conduzidos em áreas experimentais da Celulose Nipo-Brasileira S.A (CENIBRA).

Avaliação das intensidades de tráfego e carga de um Forwarder em um Latossolo Vermelho-Amarelo, situado na Região de Santa Maria de Itabira, MG.

Este estudo foi realizado em um Latossolo VermelhoAmarelo (LVA), textura argilosa, localizado no município de Santa Maria de Itabira, MG, com coordenadas $19^{\circ} 23^{\prime} 11,63 " S$ e $42^{\circ} 54^{\prime} 16,11^{\prime \prime} \mathrm{W}$ de Greenwich e altitude de 850 metros.
O preparo da área para a realização do estudo consistiu em derrubar as árvores de eucalipto com o uso de motosserra e a retirada da madeira foi feita manualmente. Em seguida, foram demarcadas para cada experimento, parcelas de quatro linhas de árvores, com 26 árvores na linha com espaçamento de $3 \times 2 \mathrm{~m}$, apresentando uma área total de $624 \mathrm{~m}^{2}$, em que dois experimentos foram conduzidos no delineamento inteiramente casualizado. No primeiro, avaliou-se o efeito da intensidade de tráfego, no qual foi utilizado um Forwarder autocarregável (marca Valmet, modelo $636 \mathrm{~S}$ ), pneus 600/55-26.5 com tara de 11,9 t, carregado com $9 \mathrm{~m}^{3}$ de madeira (densidade de $480 \mathrm{~kg} \mathrm{~m}^{-3}$ ), o qual trafegou na mesma entrelinha de acordo com os seguintes tratamentos: 1) local onde não ocorreu passadas; 2) local onde ocorreu 2 passadas; 3 ) local onde ocorreu 4 passadas; 4) local onde ocorreu 8 passadas, com uma carga de $3 / 3$ de sua capacidade, o que corresponde a $9 \mathrm{~m}^{3}$ de madeira. No segundo experimento, o Forwarder trafegou quatro vezes na mesma linha recebendo cargas correspondentes aos seguintes tratamentos: 1)local onde não ocorreu o tráfego; 2)local onde ocorreu o tráfego com $1 / 3$ de sua carga $\left.\left(3 \mathrm{~m}^{3}\right) ; 3\right)$ local onde ocorreu o tráfego com $2 / 3$ de sua carga $\left(6 \mathrm{~m}^{3}\right) ; 4$ ) local onde ocorreu o tráfego com $3 / 3$ de sua carga $\left(9 \mathrm{~m}^{3}\right)$.

Para determinar o efeito da intensidade de tráfego e carga de um Forwarder nas propriedades físicas e mecânicas do solo nos dois experimentos, coletou-se na linha de tráfego do Forwarder nas profundidades 0-3 e $10-13 \mathrm{~cm}, 10$ amostras indeformadas para cada tratamento. As amostras indeformadas foram coletadas usando um amostrador tipo Uhland com anel volumétrico de $6,40 \mathrm{~cm}$ de diâmetro e 2,54 cm de altura. Essas amostras foram parafinadas no campo, para evitar alterações na umidade a partir do momento da coleta até a realização do ensaio de compressão uniaxial no laboratório.

Avaliação das diferentes condições de camadas de resíduos sobre diferentes intensidades de tráfego de um Forwarder, em um Latossolo Amarelo e em um Latossolo Vermelho, situados nas Regiões de Belo Oriente e São João Evangelista, MG.

Este estudo foi realizado em um Latossolo Amarelo (LA), textura argilosa, localizado no município de Belo Oriente, MG, apresentando as coordenadas: $19^{\circ} 18^{\prime} 23^{\prime \prime S}$ e $42^{\circ} 25^{\prime} 31^{\prime \prime W}$ de Greenwich e altitude de 300 metros e em um Latossolo Vermelho (LV), textura muito argilosa, localizado no município de São João Evangelista, MG, apresentando as coordenadas: $18^{\circ} 34^{\prime} 12^{\prime \prime} \mathrm{S}$ e $42^{\circ} 52^{\prime} 53^{\prime \prime} \mathrm{W}$ de Greenwich e altitude de 860 metros. 
Foram demarcadas, nas Regiões de Belo Oriente e São João Evangelista, parcelas de $36 \mathrm{~m}$ de comprimento e $4 \mathrm{~m}$ de largura, o que corresponde à linha de 12 árvores de eucaliptos. A colheita florestal foi realizada manualmente, com o uso de motosserra e o baldeio da madeira foi feito com o Forwarder autocarregável (marca Valmet, modelo $636 \mathrm{~S}$ ), pneu 600/55 -26.5 com tara de 11,9 t, carregado com $9 \mathrm{~m}^{3}$ de madeira (densidade de $480 \mathrm{~kg} \mathrm{~m}^{-3}$ ), que trafegou 2 e 8 vezes em entrelinhas distintas nas diferentes condições de uso de resíduo.

Para avaliar o efeito do resíduo e o do número de passadas sobre a pressão de preconsolidação, foram coletadas nas Regiões de Belo Oriente e São João Evangelista, 20 amostras indeformadas, obedecendo ao delineamento inteiramente casualizado, na linha de tráfego do Forwarder na profundidade de $10-13 \mathrm{~cm}$, após esse ter trafegado 2 e 8 vezes nas seguintes condições de disposição dos resíduos correspondendo aos tratamentos: 1) Galhada (G);2) Galhada mais a Casca (GC); 3) Solo Sem Resíduo (SR) e 4) Testemunha (local com presença de poucos resíduos aleatórios principalmente folhas, mas sem tráfego do Forwarder (ST)). Essas amostras foram coletadas usando-se um amostrador tipo Uhland com anel volumétrico de $6,40 \mathrm{~cm}$ de diâmetro e 2,54 cm de altura, sendo parafinadas no campo para evitar alterações na umidade a partir do momento da coleta até o da realização do ensaio de compressão uniaxial no laboratório.

No laboratório, os ensaios de compressão uniaxial foram realizados de acordo com Bowles (1986), modificado por Dias Júnior \& Pierce (1995) nas amostras indeformadas, coletadas com a umidade na qual as operações com o Forwarder foram realizadas.

O ensaio de compressão uniaxial foi realizado utilizando-se um consolidômetro da marca Boart Longyear, por meio do qual as pressões foram aplicadas utilizando- se ar comprimido. As pressões aplicadas a cada amostra obedeceram à seguinte ordem: $25,50,100,200,400,800$, $1600 \mathrm{kPa}$. Cada pressão foi aplicada até que $90 \%$ da deformação máxima fosse atingida (Taylor, 1948) e, após essa condição ser atingida, aplicou-se uma nova pressão. Após a realização do ensaio de compressão uniaxial, as pressões de preconsolidação $\left(\sigma_{p}\right)$ foram obtidas na curva de compressão do solo, de acordo com Dias Júnior \& Pierce (1995).

Nas amostras indeformadas utilizadas para determinar a $\sigma_{\mathrm{p}}$, determinou-se também a umidade, a densidade do solo, o volume total de poros de acordo com Empresa Brasileira de Pesquisa Agropecuária - Embrapa (1997) para as Regiões de Santa Maria de Itabira, Belo Oriente e São João Evangelista.

Os teores de matéria orgânica (Raij \& Quaggio, 1983), textura (Day, 1986) e densidade de partículas (Blake \& Hartge, 1986), foram determinados nas amostras de solo coletadas das porções superiores e inferiores dos anéis da amostragem. Nas amostras indeformadas, também foram determinados a capacidade de campo e o ponto de murcha permanente (Klute, 1986). As características químicas dos Latossolos Vermelho-Amarelo (LVA), Latossolo Amarelo (LA) e Latossolo Vermelho (LV) foram realizadas com base nos elementos no extrato do ataque sulfúrico, segundo Embrapa (1997).

\section{RESULTADOS E DISCUSSÃO}

Os teores de matéria orgânica, textura, densidade de partículas, densidade do solo, capacidade de campo e o ponto de murcha permanente são apresentados na Tabela 1.

Os resultados das características químicas dos Latossolos Vermelho-Amarelo (LVA), Latossolo Amarelo (LA) e Latossolo Vermelho (LV) encontram-se na Tabela 2.

Tabela 1 - Características físicas de um Latossolo Vermelho-Amarelo (LVA) localizado na Região de Santa Maria de Itabira, na profundidade de 0 -3 e $10-13$ cm, de um Latossolo Amarelo (LA), localizado na Região de Belo Oriente e de um Latossolo Vermelho (LV) localizado na Região de São João Evangelista, na profundidade de 10 - $13 \mathrm{~cm}$.

\begin{tabular}{ccccccccccc}
\hline Solos & Prof. & Argila & Areia & Silte & $\mathrm{MO}^{1}$ & $\mathrm{Dp}^{1}$ & $\mathrm{Ds}^{1}$ & $\mathrm{PT}^{1}$ & $\mathrm{CC}^{1}$ & $\mathrm{PMP}^{1}$ \\
\hline \multirow{2}{*}{ LVA } & $\mathrm{cm}$ & $---------------\mathrm{g} \mathrm{kg}^{-1}-------------$ & & $-----\mathrm{g} \mathrm{cm}^{-3}----$ & $\mathrm{m}^{3} \mathrm{~m}^{3}$ & $-----\mathrm{kg} \mathrm{kg}^{-1}----$ \\
& $0-3$ & 537 & 407 & 56 & 40 & 2,50 & 0,98 & 0,61 & 0,30 & 0,26 \\
LA & $10-13$ & 570 & 367 & 63 & 25 & 2,56 & 0,94 & 0,63 & 0,31 & 0,25 \\
LV & $10-13$ & 590 & 343 & 67 & 28 & 2,56 & 1,15 & 0,55 & 0,28 & 0,23 \\
\hline
\end{tabular}

${ }^{1} \mathrm{MO}=$ matéria orgânica; $\mathrm{Dp}=$ densidade de partículas; $\mathrm{Ds}=$ densidade do solo; $\mathrm{PT}=$ volume total de poros; $\mathrm{CC}=\mathrm{Capacidade}$ de campo (amostras submetidas à tensão de $10 \mathrm{kPa}$ ); PMP = ponto de murcha permanente (amostras submetidas à tensão de $1500 \mathrm{kPa})$. 
Efeito da intensidade de tráfego do Forwarder na Região de Santa Maria de Itabira.

Na profundidade de $0-3 \mathrm{~cm}$, não foi constatado efeito da intensidade de tráfego na umidade (U), densidade do solo (Ds) e no volume total de poros (PT). Já na camada de $10-13 \mathrm{~cm}$, essas propriedades sofreram alterações, quando o solo foi submetido ao tráfego do
Forwarder, não sendo, entretanto, diferentes para as diferentes passadas (Tabela 3 ).

Avaliando o efeito da intensidade de tráfego em profundidade, nota-se (Tabela 3) que não houve diferença na $U$ entre as profundidades estudadas. A profundidade de $10-13 \mathrm{~cm}$, apresentou os maiores valores de Ds, exceto onde não houve o tráfego e os menores de PT, indicando que o tráfego alterou a estrutura do solo em profundidade.

Tabela 2 - Características químicas de um Latossolo Vermelho-Amarelo (LVA) localizado na Região de Santa Maria de Itabira, de um Latossolo Amarelo (LA) localizado na Região de Belo Oriente e um Latossolo Vermelho (LV), localizado na Região de São João Evangelista.

\begin{tabular}{ccccccc}
\hline Solos & $\mathrm{SiO}_{2}$ & $\mathrm{Al}_{2} \mathrm{O}_{3}$ & $\mathrm{Fe}_{2} \mathrm{O}_{3}$ & $\mathrm{Ki}$ & $\mathrm{Kr}$ & Classe mineralógica $^{1}$ \\
\hline LVA & $----------\mathrm{g} \mathrm{kg}^{-1}---------$ & & & & \\
LA & 71 & 173 & 61 & 0,70 & 0,57 & Oxídico-gibbsítco \\
LV & 245 & 252 & 77 & 1,65 & 1,38 & Caulinítico \\
\hline
\end{tabular}

$\mathrm{Ki}$ : relação molecular $\mathrm{SiO}_{2} / \mathrm{Al}_{2} \mathrm{O}_{3} ; \mathrm{Kr}$ : relação molecular $\mathrm{SiO}_{2} /\left(\mathrm{Al}_{2} \mathrm{O}_{3}+\mathrm{Fe}_{2} \mathrm{O}_{3}\right) .{ }^{1}$ Embrapa $(1997,2006)$.

Tabela 3 - Efeito da intensidade do tráfego de um Forwarder nas propriedades físicas e mecânicas do Latossolo Vermelho Amarelo na Região de Santa Maria de Itabira, nas profundidades de 0 -3 e $10-13$ cm.

\begin{tabular}{|c|c|c|c|c|c|}
\hline \multirow{2}{*}{$\begin{array}{c}\text { Camada } \\
(\mathrm{cm})\end{array}$} & \multicolumn{5}{|c|}{ Número de passadas do Forwarder ${ }^{1}$} \\
\hline & 0 passadas $^{+}$ & 2 passadas $^{+}$ & 4 passadas $^{+}$ & 8 passadas $^{+}$ & $\mathrm{CV}^{*}(\%)$ \\
\hline \multicolumn{6}{|c|}{$\mathrm{U}\left(\mathrm{kg} \mathrm{kg}^{-1}\right)$} \\
\hline $0-3$ & 0,2638 a A & 0,2268 a A & 0,2373 a A & 0,2399 a A & 14,63 \\
\hline $10-13$ & 0,2613 a $A$ & $0,2308 \mathrm{~b} \mathrm{~A}$ & $0,2237 \mathrm{~b} \mathrm{~A}$ & $0,2207 \mathrm{~b} \mathrm{~A}$ & 10,21 \\
\hline $\mathrm{CV}^{*}(\%)$ & 18,72 & 3,62 & 10,45 & 10,49 & \\
\hline \multicolumn{6}{|c|}{ Ds $\left(\mathrm{kg} \mathrm{dm}^{-3}\right)$} \\
\hline $0-3$ & 0,98 a $\mathrm{A}$ & 1,00 a B & 0,99 a B & 1,00 a B & 8,46 \\
\hline $10-13$ & 0,94 b B & 1,08 a $\mathrm{A}$ & 1,14 a A & 1,11 a $\mathrm{A}$ & 5,91 \\
\hline $\mathrm{CV} *(\%)$ & 3,96 & 4,66 & 8,86 & 9,18 & \\
\hline \multicolumn{6}{|c|}{ PT $\left(\mathrm{m}^{3} \mathrm{~m}^{-3}\right)$} \\
\hline $0-3$ & 0,6101 a B & 0,5982 a A & 0,6003 a $\mathrm{A}$ & 0,5995 a A & 5,55 \\
\hline $10-13$ & 0,6335 a A & $0,5788 \mathrm{~b} \mathrm{~B}$ & 0,5539 b B & $0,5680 \mathrm{~b} \mathrm{~A}$ & 4,18 \\
\hline $\mathrm{CV}^{*}(\%)$ & 2,34 & 3,19 & 6,44 & 6,59 & \\
\hline \multicolumn{6}{|c|}{$\sigma_{\mathrm{p}}(\mathrm{kPa})$} \\
\hline $0-3$ & 221 c A & 295 b B & 340 a $\mathrm{A}$ & $303 \mathrm{~b} \mathrm{~A}$ & 12,12 \\
\hline $10-13$ & $228 \mathrm{~b} \mathrm{~A}$ & 360 a A & 346 a A & 334 a A & 10,42 \\
\hline $\mathrm{CV}^{*}(\%)$ & 20,66 & 7,80 & 7,94 & 10,42 & \\
\hline
\end{tabular}

${ }^{1} \mathrm{U}=$ Umidade; Ds = densidade do solo; PT = volume total de poros; $\sigma_{\mathrm{p}}=$ pressão de preconsolidação, $*$ CV = Coeficiente variação. Médias seguidas da mesma letra minúscula na linha não diferem entre si, quanto ao número de passadas do Forwarder na mesma profundidade, pelo teste de Scott-Knott $(\mathrm{p}<0,05)$. Médias seguidas da mesma letra maiúscula não diferem entre si, quanto à profundidade no mesmo número de passadas do Forwarder, pelo teste de Scott-Knott $(\mathrm{p}<0,05)$ (+ Média de 10 repetições por profundidade). 
Esses resultados concordam com os obtidos por Miranda et al. (2003), que observou o efeito da compactação causado pelo tráfego de um trator agrícola na profundidade 10 a $20 \mathrm{~cm}$ e com os obtidos por Silva et al. (2007), segundo o qual a camada compactada localizou-se na profundidade de 5 a $25 \mathrm{~cm}$ em um Latossolo Amarelo.

As pressões de preconsolidação $\left(\sigma_{\mathrm{p}}\right)$, da profundidade de $0-3 \mathrm{~cm}$ foram diferentes estatisticamente nas diferentes intensidade de tráfego. Observa-se que, para 4 passadas do Forwarder ocorreu o maior valor $\sigma_{\mathrm{p}}$, seguidas das condições de 2 e 8 passadas, as quais não diferiram entre si. O menor valor de $\sigma_{\mathrm{p}}$ ocorreu onde não houve o tráfego, evidenciando que o solo suportou menores pressões no passado do que as aplicadas pelo Forwarder. Já na profundidade de $10-13 \mathrm{~cm}$, não houve diferenças estatísticas entre as condições de intensidade de tráfego, entretanto, essas foram diferentes estatisticamente das $\sigma_{\text {p }}$ do local onde não houve tráfego (Tabela 3). Como as $\sigma_{\mathrm{p}}$ induzidas na profundidade $10-13 \mathrm{~cm}$ foram independentes do número de passadas e o número de passadas igual a 4 foi o que causou maior compactação na profundidade de $0-3 \mathrm{~cm}$, identificou-se, o número de passadas igual a 4 como sendo o limite para a degradação da estrutura do solo.

Em profundidade, observou-se diferença estatística na $\sigma_{\mathrm{p}}$ somente quando o tráfego foi realizado 2 vezes na mesma entrelinha (Tabela 3 ), sendo a profundidade de $10-13 \mathrm{~cm}$ a que apresentou o maior valor de $\sigma_{\mathrm{p}}$, indicando um efeito mais pronunciado da compactação do solo em profundidade nas primeiras passadas do Forwarder. Esses resultados corroboram com os obtidos por SchackKirchner et al. (2007) que verificou em um estudo de tráfego controlado, que as maiores deformações no solo ocorrem logo após a primeira passagem de um trator florestal, e também corroboram com os de Silva et al. (2007), os quais relataram que as repetidas passadas dos rodados aumentam a degradação da estrutura em profundidade.

Efeito da carga de tráfego do Forwarder na Região de Santa Maria de Itabira.

A Ds, U, e PT não apresentaram diferenças significativas na profundidade de $0-3 \mathrm{~cm}$, quando o tráfego do Forwarder foi realizado com diferentes cargas (Tabela 4). Na profundidade 10-13 cm, verificou-se que o tráfego do Forwarder com diferentes cargas elevou significativamente os valores da Ds e reduziu significativamente os valores de U e PT em relação aos valores da condição onde não houve tráfego do Forwarder, não havendo, entretanto, diferenças significativas entre os valores de Ds, U e PT com a utilização de diferentes cargas $1 / 3\left(3 \mathrm{~m}^{3}\right), 2 / 3\left(6 \mathrm{~m}^{3}\right)$ e $3 / 3\left(9 \mathrm{~m}^{3}\right)$ da carga.
O tráfego do Forwarder com diferentes cargas não causou diferença entre os valores de $\mathrm{U}$ em profundidade, enquanto que os valores da Ds e da PT apresentaram diferenças significativas onde não houve o tráfego e quando o Forwarder trafegou com $2 / 3$ de sua carga. Os valores de Ds foram maiores e os de PT foram menores na profundidade de $10-13 \mathrm{~cm}$ (Tabela 4), sugerindo ser essa a carga crítica para o processo de compactação.

Os valores $\sigma_{\mathrm{p}}$ na profundidade de 0-3 aumentaram significativamente quando o Forwarder trafegou com as diferentes cargas em relação ao local onde não houve o tráfego (Tabela 4). Os valores de $\sigma$ não diferiram entre si para as diferentes cargas do Forwarder. Na profundidade de $10-13 \mathrm{~cm}$, os maiores valores de $\sigma_{\mathrm{p}}$ ocorreram para cargas de $2 / 3\left(6 \mathrm{~m}^{3}\right)$ e $3 / 3\left(9 \mathrm{~m}^{3}\right)$, sugerindo novamente, ser a carga 2/3 a crítica para o processo de compactação do solo. Esses resultados corroboram com os de Horn \& Lebert (1994), que observaram que a aplicação de cargas dinâmicas no solo pode causar compactação em diferentes profundidades do solo.

Avaliação das diferentes condições de camadas de resíduos sobre diferentes intensidades de tráfego de um Forwarder, em um Latossolo Amarelo e em um Latossolo Vermelho, situados nas Regiões de Belo Oriente e São João Evangelista, MG.

Na Região de Belo Oriente, a utilização de Galhada mais Casca (GC) e Galhada (G) promoveu aumentos significativos na $U$ quando o Forwarder trafegou 2 e 8 vezes, em comparação com o local onde não foi utilizado o resíduo (SR) e onde não houve tráfego (ST) (Tabela 5). Com o aumento da intensidade de tráfego para 8 passadas, as condições de resíduos foram diferentes, sendo a condição GC a que apresentou a maior umidade, seguida da condição $\mathrm{G}$, indicando uma preservação da água do solo nessas condições. A umidade do solo nas diferentes condições de resíduos não foi diferente para 2 e 8 passadas. Na Região de São João Evangelista, entretanto, as U não diferiram estatisticamente entre as condições de disposição dos resíduos e nas intensidades de tráfego (Tabela 5), devido, provavelmente, aos maiores teores de argila e matéria orgânica, encontrados no LV em relação ao LA, aumentado assim à retenção de água.

A Ds não foi influenciada pelas condições de disposição dos resíduos nas duas regiões, já a intensidade de tráfego alterou significativamente os valores de Ds somente na Região de Belo Oriente (Tabela 5). Dessa forma, nota-se que a ausência de tráfego e a presença de resíduos apresentaram, em ambas as regiões, valores de densidades do solo estatisticamente inferiores aos valores encontrados 
na condição sem a presença dos resíduos (SR), sugerindo preservação da estrutura do solo devido à dissipação das pressões aplicadas pelos resíduos. Em uma mesma condição de resíduo, e quando o número de passadas do Forwarder aumentou de 2 para 8 (Tabela 5), houve um aumento significativo nos valores das densidades do solo somente na região do Belo Oriente, sugerindo ser o Latossolo Amarelo dessa região mais susceptível à compactação do que o Latossolo Vermelho da Região de São João Evangelista. Esses maiores valores de Ds vêm ao encontro dos resultados de Ferreira et al. (1999) e Ajayi et al. (2009) os quais solos cauliniticos (Tabela 2) implicam o desenvolvimento de macroestrutura do tipo em blocos, promovendo um encaixe face a face dos blocos originando latossolos com maior densidade do solo, sendo também esses valores aumentados quando os mesmos são submetidos a um maior número de passadas dos equipamentos corroborando com os resultados de Silva et al. (2007).

A PT teve comportamento inverso ao da Ds (Tabela 5), corroborando com observações anteriores de que a compactação do solo intensificou-se mais com a realização do tráfego, em condições de Solo Sem Resíduo (SR).

As $\sigma_{\mathrm{p}}$ aumentaram significativamente quando o tráfego foi realizado em condição de SR em ambas as regiões (Tabela 5), sendo essa condição a mais crítica em relação à compactação devido aos maiores valores de $\sigma_{\mathrm{p}}$. As $\sigma_{\mathrm{p}}$ das condições GC e G para 2 passadas não diferiram estatisticamente na Região de Belo Oriente. Tanto na Região do Belo Oriente para 8 passadas, quanto na Região de São João Evangelista para 2 e 8 passadas, a condição $\mathrm{GC}$ foi a que apresentou menores valores de $\sigma_{\mathrm{p}}$ (Tabela 5). Os menores valores da $\sigma_{\mathrm{p} \text {, }}$ encontrados nas condições de GC e G, indicam uma distribuição das pressões aplicadas pelo Forwarder através dos resíduos, resultando em menores $\sigma_{\mathrm{p}}$ induzidas aos solos, sugerindo que as operações florestais sejam realizadas sobre os resíduos, visando minimizar os efeitos da compactação do solo. Esses resultados concordam com os obtidos por (Seixas et al., 1998; Silva et al., 2007) os quais detectaram reduções significativas da compactação do solo devido ao tráfego realizado sobre camadas de resíduos.

Tabela 4 - Efeito da carga de tráfego do Forwarder nas propriedades físicas e mecânicas do Latossolo Vermelho Amarelo na Região de Santa Maria de Itabira, nas profundidades de 0-3 e $10-13$ cm.

\begin{tabular}{|c|c|c|c|c|c|}
\hline \multirow[b]{2}{*}{$\begin{array}{l}\text { Camada } \\
(\mathrm{cm})\end{array}$} & \multicolumn{5}{|c|}{ Carga do Forwarder } \\
\hline & $\begin{array}{c}0 \text { carga }^{+} \\
-\end{array}$ & $\begin{array}{c}1 / 3 \text { carga }^{+} \\
3 \mathrm{~m}^{3}\end{array}$ & $\begin{array}{c}2 / 3 \text { carga }^{+} \\
6 \mathrm{~m}^{3}\end{array}$ & $\begin{array}{c}3 / 3 \text { carga }^{+} \\
9 \mathrm{~m}^{3}\end{array}$ & $\mathrm{CV}^{*}(\%)$ \\
\hline \multicolumn{6}{|c|}{$\mathrm{U}\left(\mathrm{kg} \mathrm{kg}^{-1}\right)$} \\
\hline $0-3$ & 0,2638 a $A$ & 0,2490 a A & 0,2475 a A & 0,2485 a A & 15,39 \\
\hline $10-13$ & 0,2613 a $A$ & $0,2263 \mathrm{~b} A$ & $0,2309 \mathrm{~b} \mathrm{~A}$ & $0,2327 \mathrm{~b} A$ & 9,87 \\
\hline $\mathrm{CV} *(\%)$ & 18,72 & 12,48 & 8,66 & 8,22 & \\
\hline \multicolumn{6}{|c|}{ Ds $\left(\mathrm{kg} \mathrm{dm}^{-3}\right)$} \\
\hline $0-3$ & 0,98 a $\mathrm{A}$ & 1,00 a $\mathrm{A}$ & 1,01 a B & 0,99 a $\mathrm{A}$ & 9,17 \\
\hline $10-13$ & 0,94 b B & 1,08 a $\mathrm{A}$ & 1,13 a $\mathrm{A}$ & 1,06 a $\mathrm{A}$ & 6,57 \\
\hline $\mathrm{CV}^{*}(\%)$ & 3,96 & 10,45 & 6,82 & 8,48 & \\
\hline \multicolumn{6}{|c|}{$\mathrm{PT}\left(\mathrm{m}^{3} \mathrm{~m}^{-3}\right)$} \\
\hline $0-3$ & 0,6101 a B & 0,6006 a $A$ & 0,5971 a A & 0,6055 a A & 5,94 \\
\hline $10-13$ & 0,6335 a A & 0,5772 b A & $0,5590 \mathrm{~b}$ B & $0,5850 \mathrm{~b} \mathrm{~A}$ & 4,59 \\
\hline $\mathrm{CV}^{*}(\%)$ & 2,34 & 7,24 & 4,98 & 5,75 & \\
\hline \multicolumn{6}{|c|}{$\sigma_{\mathrm{p}}(\mathrm{kPa})$} \\
\hline $0-3$ & $221 \mathrm{~b} \mathrm{~A}$ & 312 a A & 336 a A & 349 a A & 12,08 \\
\hline $10-13$ & 228 c A & 296 b A & 354 a A & 335 a A & 12,45 \\
\hline $\mathrm{CV}^{*}(\%)$ & 20,66 & 12,56 & 9,38 & 8,79 & \\
\hline
\end{tabular}

${ }^{1} \mathrm{U}=$ Umidade; $\mathrm{Ds}=$ densidade do solo; $\mathrm{PT}=$ volume total de poros; $\sigma_{\mathrm{p}}=$ pressão de preconsolidação, * $\mathrm{CV}=$ Coeficiente variação. Médias seguidas da mesma letra minúscula na linha não diferem entre si, quanto à carga do Forwarder na mesma profundidade, pelo teste de Scott-Knott $(\mathrm{p}<0,05)$. Médias seguidas da mesma letra maiúscula não diferem entre si, quanto à profundidade na mesma carga do Forwarder, pelo teste de Scott-Knott $(\mathrm{p}<0,05)$ (+Médias de 10 repetições por profundidade). 
Tabela 5 - Propriedades físicas e mecânicas de um Latossolo Amarelo da Região de Belo Oriente, MG e de um Latossolo Vermelho da Região de São João Evangelista, MG na profundidade de 10 - 13 cm, sob diferentes condições de resíduos e intensidades de tráfego.

\begin{tabular}{|c|c|c|c|c|c|c|}
\hline \multirow{2}{*}{ Regiões } & \multirow{2}{*}{$\mathrm{N}^{\circ}$ passadas do Forwarder } & \multicolumn{5}{|c|}{ Condições de disposição dos resíduos ${ }^{1}$} \\
\hline & & $\mathrm{ST}^{+}$ & $\mathrm{GC}^{+}$ & $\mathrm{G}^{+}$ & $\mathrm{SR}^{+}$ & $\mathrm{CV}^{*}(\%)$ \\
\hline \multirow{16}{*}{ Belo Oriente } & \multicolumn{6}{|c|}{$\mathrm{U}\left(\mathrm{kg} \mathrm{kg}^{-1}\right)$} \\
\hline & 2 passadas & $0,2510 \mathrm{~b} \mathrm{~A}$ & 0,2716 a A & 0,2684 a A & $0,2514 \mathrm{~b} \mathrm{~A}$ & 5,52 \\
\hline & 8 passadas & 0,2510 c A & 0,2717 a A & $0,2604 \mathrm{~b} \mathrm{~A}$ & 0,2473 c A & 4,55 \\
\hline & $\mathrm{CV}^{*}(\%)$ & 3,16 & 5,87 & 5,68 & 4,84 & \\
\hline & \multicolumn{6}{|c|}{ Ds $\left(\mathrm{kg} \mathrm{dm}^{-3}\right)$} \\
\hline & 2 passadas & $1,15 \mathrm{c} \mathrm{A}$ & $1,22 \mathrm{~b} \mathrm{~B}$ & $1,23 \mathrm{~b} \mathrm{~B}$ & 1,29 a B & 3,60 \\
\hline & 8 passadas & $1,15 \mathrm{c} \mathrm{A}$ & $1,26 \mathrm{~b} \mathrm{~A}$ & $1,28 \mathrm{~b} \mathrm{~A}$ & 1,34 a $\mathrm{A}$ & 3,30 \\
\hline & $\mathrm{CV}^{*}(\%)$ & 2,32 & 4,16 & 4,12 & 2,70 & \\
\hline & \multicolumn{6}{|c|}{ PT $\left(\mathrm{m}^{3} \mathrm{~m}^{-3}\right)$} \\
\hline & 2 passadas & 0,5523 a A & $0,5262 \mathrm{~b} \mathrm{~A}$ & $0,5203 \mathrm{~b} \mathrm{~A}$ & 0,4942 с A & 3,33 \\
\hline & 8 passadas & 0,5523 a A & $0,5064 \mathrm{~b} \mathrm{~B}$ & 0,5006 b B & 0,4789 с В & 3,20 \\
\hline & $\mathrm{CV}^{*}(\%)$ & 1,85 & 3,99 & 3,98 & 2,88 & \\
\hline & \multicolumn{6}{|c|}{$\sigma_{\mathrm{p}}(\mathrm{kPa})$} \\
\hline & 2 passadas & $125 \mathrm{c} \mathrm{A}$ & $205 \mathrm{~b} \mathrm{~B}$ & $210 \mathrm{~b} \mathrm{~B}$ & 264 a B & 10,12 \\
\hline & 8 passadas & $125 \mathrm{~d} A$ & 228 с A & $264 \mathrm{~b} \mathrm{~A}$ & 314 a A & 10,06 \\
\hline & $\mathrm{CV}^{*}(\%)$ & 9,50 & 10,39 & 10,27 & 9,05 & \\
\hline \multirow{16}{*}{$\begin{array}{c}\text { São João } \\
\text { Evangelista }\end{array}$} & \multicolumn{6}{|c|}{$\mathrm{U}\left(\mathrm{kg} \mathrm{kg}^{-1}\right)$} \\
\hline & 2 passadas & 0,3548 a A & 0,3721 a $\mathrm{A}$ & 0,3553 a $\mathrm{A}$ & 0,3419 a $\mathrm{A}$ & 12,70 \\
\hline & 8 passadas & 0,3548 a A & 0,3642 a A & 0,3769 a A & 0,3563 a A & 8,87 \\
\hline & $\mathrm{CV}^{*}(\%)$ & 6,93 & 12,76 & 9,53 & 13,22 & \\
\hline & \multicolumn{6}{|c|}{ Ds $\left(\mathrm{kg} \mathrm{dm}^{-3}\right)$} \\
\hline & 2 passadas & 0,86 с A & 0,96 b A & 0,96 b A & 1,05 a $\mathrm{A}$ & 8,18 \\
\hline & 8 passadas & 0,86 с A & 0,96 b A & $0,98 \mathrm{~b} \mathrm{~A}$ & 1,02 a $A$ & 5,21 \\
\hline & $\mathrm{CV}^{*}(\%)$ & 3,82 & 9,56 & 5,99 & 6,39 & \\
\hline & \multicolumn{6}{|c|}{$\mathrm{PT}\left(\mathrm{m}^{3} \mathrm{~m}^{-3}\right)$} \\
\hline & 2 passadas & 0,6585 a $\mathrm{A}$ & $0,6218 \mathrm{~b} \mathrm{~A}$ & $0,6201 \mathrm{~b} \mathrm{~A}$ & 0,5872 с A & 4,96 \\
\hline & 8 passadas & 0,6585 a $A$ & $0,6204 \mathrm{~b} \mathrm{~A}$ & $0,6131 \mathrm{~b} \mathrm{~A}$ & 0,5959 с A & 3,16 \\
\hline & $\mathrm{CV}^{*}(\%)$ & 1,97 & 5,85 & 3,72 & 4,35 & \\
\hline & \multicolumn{6}{|c|}{$\sigma_{\mathrm{p}}(\mathrm{kPa})$} \\
\hline & 2 passadas & $125 \mathrm{~d} \mathrm{~A}$ & 144 c A & $161 \mathrm{~b} \mathrm{~B}$ & 222 a B & 15,18 \\
\hline & 8 passadas & $125 \mathrm{~d} \mathrm{~A}$ & $152 \mathrm{c} \mathrm{A}$ & 192 b A & 248 a $\mathrm{A}$ & 14,44 \\
\hline & $\mathrm{CV}^{*}(\%)$ & 8,50 & 11,28 & 13,82 & 16,92 & \\
\hline
\end{tabular}

${ }^{1}$ Condições de disposição dos resíduos: $\mathrm{ST}$ = sem tráfego do forwarder; $\mathrm{GC}=$ uso da Galhada + Casca; $\mathrm{G}=$ uso somente da galhada; $\mathrm{SR}=$ Solo Sem Resíduo; *CV = coeficiente de variação. U = Umidade; Ds = densidade do solo; PT = volume total de poros; $\sigma_{\mathrm{p}}=$ pressão de preconsolidação. Médias seguidas da mesma letra minúscula na linha não diferem entre si, quanto à condição de disposição do resíduo no mesmo número de passadas do Forwarder, pelo teste de Scott-Knott ( $<<0,05)$. Médias seguidas da mesma letra maiúscula não diferem entre si, quanto ao número de passadas do Forwarder na mesma condição de disposição dos resíduos, pelo teste de Scott-Knott $(\mathrm{p}<0,05)$ (+Médias de 20 repetições). 
Analisando o número de passadas em relação a cada condição (Tabela 5), observa-se que à medida que a intensidade de tráfego aumentou para 8 passadas, houve um aumento significativo nos valores de $\sigma_{\mathrm{p},}$ nas condições de GC, G e SR exceto na Região de São João Evangelista, para a condição GC. Respostas semelhantes foram encontradas por (Wood et al., 1993; Silva et al., 2007), que verificaram que o número crescente de passadas aumenta a compactação do solo.

\section{CONCLUSÕES}

O tráfego do Forwarder causou compactação nos solos LVA na Região de Santa Maria de Itabira, no LA na Região de Belo Oriente e no LV na Região de São João Evangelista.

A compactação do solo aumentou com o aumento da intensidade de tráfego.

As condições de Galhada + Casca (GC) e Galhada (G) minimizaram o efeito da compactação do solo.

\section{REFERÊNCIAS BIBLIOGRÁFICAS}

AJAYI, A.E.; DIAS JUNIOR, M.S.; CURI, N.; ARAUJO JUNIOR, C.F.; SOUZA, T.T.T.; INDA JUNIOR, A.V. Strength attibutes and compaction susceptibility of Brazilian Latossols. Soil and Tillage Research, Amsterdam, n.1, p.122-127, set. 2009.

BLAKE, G.R.; HARTGE, K.H. Particle density. In: KLUTE, C. (Ed.). Methods of soil analysis: part 1, physical and mineralogical methods. 2.ed. Madison: American Society Agronomy, 1986. p.377-382. (Agronomy Monograph, 9).

BOWLES, J.E. Engieneering properties of soils and their measurements. 3.ed. Auckland: McGraw-Hill, 1986. 218p.

DAY, P.R. Particle fractionation and particle size analysis. In: KLUTE, C.A. (Ed.). Methods of soil analysis: part 1, physical and mineralogical methods. 2.ed. Madison: American Society Agronomy, 1986. p.545-567. (Agronomy Monograph, 9).

DIAS JUNIOR, M.S.; LEITE, F.P.; LASMAR JUNIOR, E.; ARAÚJO JUNIOR, C.F. Traffic effects on the soil preconsolidation pressure due to eucalyptus harvest operations. Scientia Agricola, Piracicaba, v.62, n.3, p.248-255, maio/jun. 2005.

DIAS JUNIOR, M.S.; PIERCE, F.J. Revisão de literatura: o processo de compactação do solo e sua modelagem.
Revista Brasileira de Ciência do Solo, Campinas, v.20, n.2, p.175-182, maio/ago. 1996.

DIAS JUNIOR, M.S.; PIERCE, F.J. A simple procedure for estimating preconsolidation pressure from soil compression curves. Soil Technology, Amsterdam, v.8, n.2, p.139-151, Nov. 1995.

DIAS JUNIOR, M.S.; SILVA, S.R.; SANTOS, N.S.; ARAUJO-JUNIOR, C.F. Assessment of the soil compaction of two ultisols caused by logging operations. Revista Brasileira de Ciência do Solo, Viçosa, v.32 p.2245-2253, nov./dez. 2008.

\section{EMBRAPA BRASILEIRA DE PESQUISA}

AGROPECUÁRIA. Centro Nacional de Pesquisa de Solos. Manual de métodos de análise de solo. 2.ed. Rio de Janeiro: Ministério da Agricultura e do Abastecimento, 1997. 212p.

\section{EMBRAPA BRASILEIRA DE PESQUISA}

AGROPECUÁRIA. Centro Nacional de Pesquisa de

Solos. Sistema brasileiro de classificação de solos. 2.ed.

Rio de Janeiro: Ministério da Agricultura e do

Abastecimento, 2006. 306p.

FERREIRA, M.M.; FERNANDES, B.; CURI, N. Influência da mineralogia da fração argila nas propriedades físicas de Latossolos da região sudeste do Brasil. Revista Brasileira de Ciência do Solo, Viçosa, n.3 p.515-524, jul./set. 1999.

HAMZA, M.A.; ANDERSON, W.K. Soil compaction in cropping systems a review of the nature, causes and possible solutions. Soil \& Tillage Research, Amsterdam, v.82, n.2, p.121-145, June 2005.

HORN, R.; LEBERT, M. Soil compactibility and compressibility. In: SOANE, B.D.; OUWEKERK, C. van (Eds.). Soil compaction in crop production. Amsterdam: Elsevier, 1994. p.45-69. (Developments in Agricultural Engineering, 11).

HORN, R.; VOSSBRINK, J.; BECKER, S. Modern foresty vehicles and their impacts on soil physical properties. Soil \& Tillage Research, Amsterdam, v.79, n.2, p.207219, Dec. 2004.

KLUTE, A. Water retention: laboratory methods. In: . Methods of soil analysis: part 1. 2.ed. Madison: American Society of Agronomy, 1986. p.635-686. 
LEBERT, M.; HORN, R. A method to predict the mechanical strength of agricultural soils. Soil \& Tillage Research, Amsterdam, v.19, n.2/3, p.274-286, Feb. 1991.

MARCHÃO, R.L.; BALBINO, L.C.; SILVA, E.M.; SANTOS JUNIOR, J.D.G.; AS, M.A.C.; VILELA, L.; BECQUER, T. Qualidade física de um Latossolo Vermelho sob sistemas de integração lavoura-pecuária no Cerrado. Pesquisa Agropecuária Brasileira, Brasília, n.6, p.873-882, jun. 2007.

MIRANDA, E.É.V.; DIAS JUNIOR, M.S.; GUIMARÃES, P.T.G.; PINTO, J.A.O.; ARAUJO JUNIOR, C.F.; LASMAR JUNIOR, E. Efeito do manejo e do tráfego nos modelos de sustentabilidade da estrutura de um Latossolo Vermelho cultivado com cafeeiro. Ciência e Agrotecnologia, Lavras, p.1506-1515, 2003. Edição especial.

MODOLO, A.J.; FERNANDES, H.C.; SCHAEFER, C.E.G.; SILVEIRA, J.C.M.de. Efeito da compactação do solo sobre a emergência de plântulas de soja em sistema plantio direto. Ciência e Agrotecnologia, Lavras, v. 32, n.4, p. 1259-1265, jul./ago., 2008.

RAIJ, B. van; GUAGGIO, J.A. Métodos de análise de solo para fins de fertilidade. Campinas: Instituto Agronômico, 1983. 16p. (Circular, 63).

RAPER, R.L. Agricultural traffic impacts on soil. Journal of Terramechanics, Oxford, v.42, n.3/4, p.259-280, July/ Oct. 2005.

SCHAFFER, B.; STAUBER, M.; MUELLER, T.L.; MULLER, R.; SCHULIN, R. Soil and macro-pores under uniaxial compression: I mechanical stability of repacked soil and deformation of different types of macro-pores. Geoderma, Amsterdam, n.1/2, p.183-191, July 2008.
SCHACK-KIRCHNER, H.; FENNER, P.T.; HILDEBRAND, E. Differentiated response of bulk density and saturated water conductivity to soil deformation by logging machines on a Ferralsol under native forest. European Journal of Soil Science, v.23, p.286-293, 2007.

REICHERT, J.M.; SUZUKI, L.E.A.S.; REINERT, D.J.; HORN, R.; HAKANSSON, I. Reference bulk density and critical degree-of-compactness for no-till crop production in subtropical highly weathered soils. Soil and Tillage Research, Amsterdam, n.2, p.242-254, Mar. 2009

SEIXAS, F.; OLIVEIRA JÚNIOR, E.D.; SOUZA, C.R. Efeito da camada de resíduos florestais na compactação do solo causada pelo transporte primário da madeira. Scientia Forestalis, Piracicaba, n.54, p.9-16, dez. 1998.

SILVA, A.R.; DIAS JUNIOR, M.S.; LEITE, F.P. Camadas de resíduos florestais e pressão de preconsolidação de dois latossolos. Pesquisa Agropecuária Brasileira, Brasília, n.1, p.89-93, jan. 2007.

SILVA, S.R.; BARROS, N.F.; COSTA, L.M.; MENDONÇA, E.S.; LEITE, P.L. Alterações do solo influenciadas pelo tráfego e carga de um "Forwarder" nas entrelinhas de uma floresta de eucalipto. Revista Brasileira de Ciência do Solo, Viçosa, n.2, p.371-377, 2007.

SUZUKI, L.E.A.S.; REICHERT, J.M.; REINERT, D.J.; LIMA, C.L.R. Grau de compactação, propriedades físicas e rendimento de culturas em Latossolo e Argissolo. Pesquisa agropecuária Brasileira, Brasília, n.8, p.11591167, ago. 2007.

TAYLOR, D.W. Fundamentals of soil mechanics. New York: J.Wiley, 1948. 700p. 